\title{
Editorial
}

\section{Low Back Pain}

\section{Seiji Ohtori, ${ }^{1}$ Yasuchika Aoki, ${ }^{2}$ Gen Inoue, ${ }^{3}$ Laura S. Stone, ${ }^{4}$ and Giustino Varrassi ${ }^{5}$}

${ }^{1}$ Department of Orthopaedic Surgery, Graduate School of Medicine, Chiba University, 1-8-1 Inohana, Chuo-ku, Chiba 260-8670, Japan

${ }^{2}$ Department of Orthopaedic Surgery, Sakura Medical Center, Toho University, 654-1 Shimoshizu, Sakura, Chiba, 285-8741, Japan

${ }^{3}$ Department of Orthopaedic Surgery, School of Medicine, Kitasato University, Kitasato 1-15-1, Sagamihara 252-0375, Japan

${ }^{4}$ The Alan Edwards Centre for Research on Pain, Faculty of Dentistry, McGill University, 740 Dr. Penfield Avenue Suite 3200, Montreal, QC, Canada H3G OG1

${ }^{5}$ Universita degli Studi dell'Aquila, 67100 L'Aquila, Italy

Correspondence should be addressed to Seiji Ohtori, sohtori@faculty.chiba-u.jp

Received 23 February 2012; Accepted 23 February 2012

Copyright (C) 2012 Seiji Ohtori et al. This is an open access article distributed under the Creative Commons Attribution License, which permits unrestricted use, distribution, and reproduction in any medium, provided the original work is properly cited.

Low back pain (LBP) is a common clinical problem and is of major socioeconomic importance. However, at the current time, there is little information about the pathogenesis of this disease. Furthermore, treatment option of LBP has not been fully explored. We focused on articles that will stimulate the continuing efforts to understand the pathology, epidemiology, diagnosis, and method of treatment of LBP.

We prepared 7 articles in this special issue as follows: "A novel image-guided, automatic high-intensity neurostimulation device for the treatment of nonspecific low back pain" by E. Schiff et al., "Evaluation of diagnosis techniques used for spinal injury related back pain" by E. Janssen et al., "Prevalence, spinal alignment, and mobility of lumbar spinal stenosis with or without chronic low back pain: a community-dwelling study" by Y. Kasukawa et al., "Assessment and treatment of abuse risk in opioid prescribing for chronic pain" by Serraillier et al., "Pain-related fear: a critical review of the related measures" by Verbunt et al., "The influence of pain distribution on walking velocity and horizontal ground reaction forces in patients with low back pain" by Etnyre et al.," Stop using the modified work apgar to measure job satisfaction" by DeVellis et al.

We believe that these 7 articles help readers to understand the mechanism and therapy of chronic LBP.

Seiji Ohtori

Yasuchika Aoki

Gen Inoue

Laura S. Stone

Giustino Varrassi 


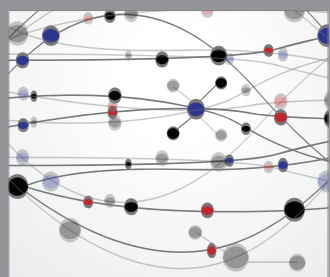

The Scientific World Journal
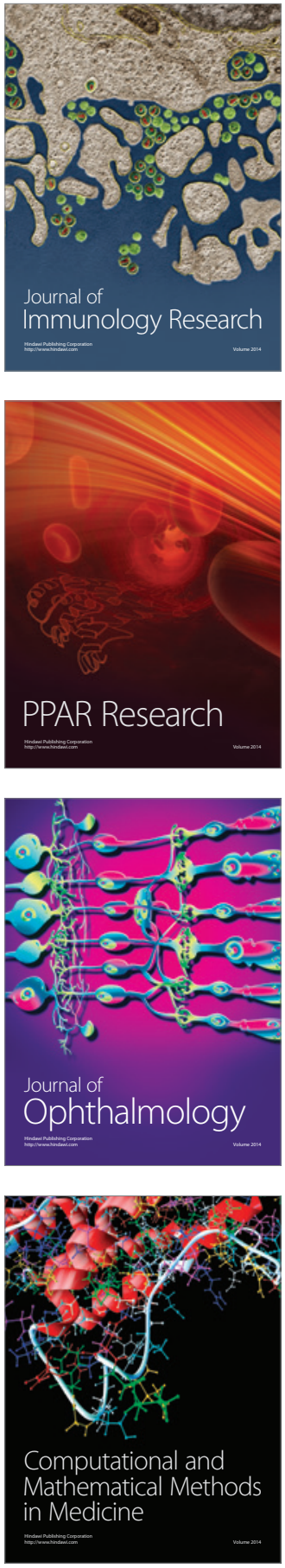

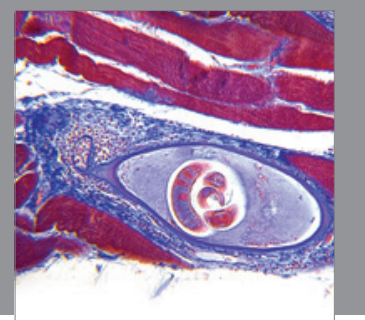

Gastroenterology

Research and Practice
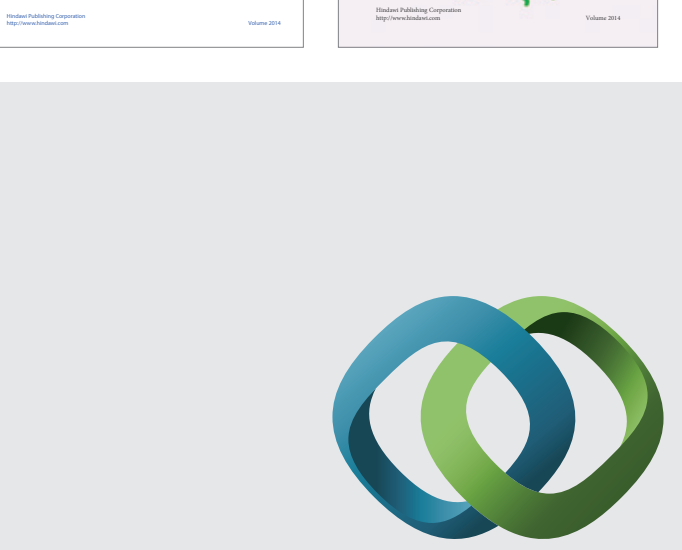

\section{Hindawi}

Submit your manuscripts at

http://www.hindawi.com
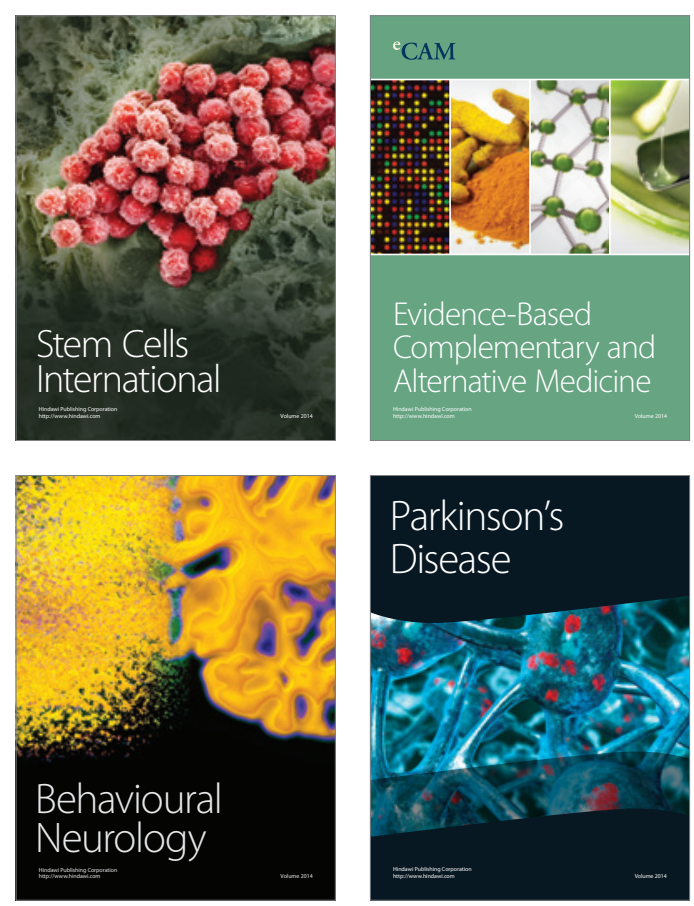

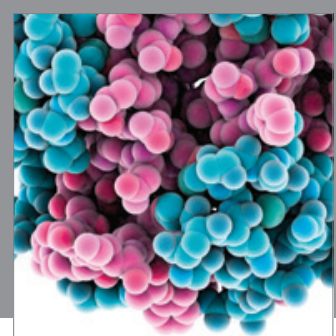

Journal of
Diabetes Research

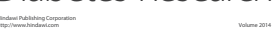

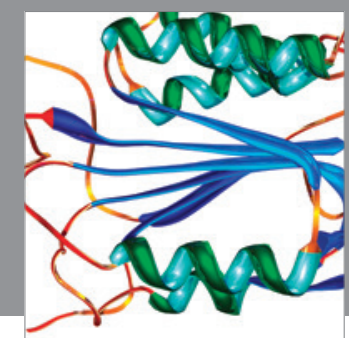

Disease Markers
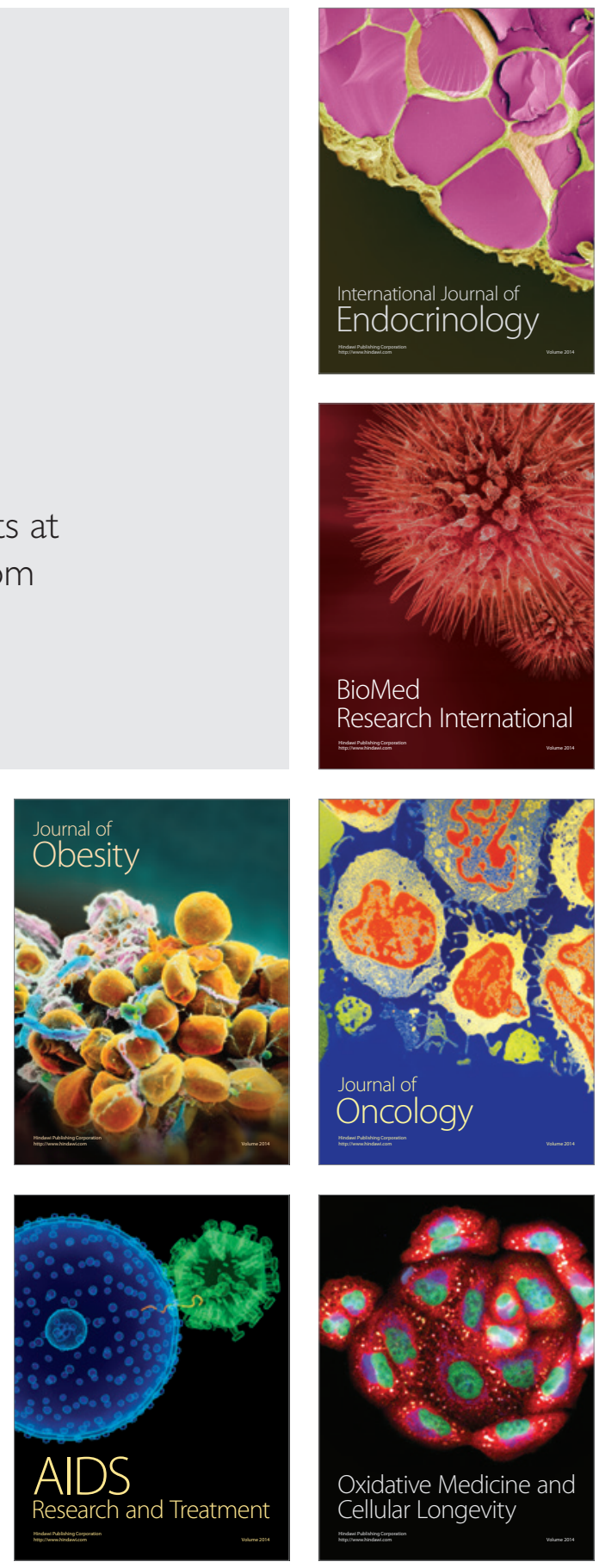